\title{
An in vitro model to evaluate the properties of matrices produced by fibroblasts from osteogenesis imperfecta and Ehlers-Danlos Syndrome patients
}

Citation for published version (APA):

Micha, D., Pals, G., Smit, T., \& Ghazanfari, S. (2020). An in vitro model to evaluate the properties of matrices produced by fibroblasts from osteogenesis imperfecta and Ehlers-Danlos Syndrome patients. Biochemical and Biophysical Research Communications, 521(2), 310-317. https://doi.org/10.1016/j.bbrc.2019.09.081

Document status and date:

Published: 08/01/2020

DOI:

10.1016/j.bbrc.2019.09.081

Document Version:

Publisher's PDF, also known as Version of record

\section{Document license:}

Taverne

Please check the document version of this publication:

- A submitted manuscript is the version of the article upon submission and before peer-review. There can be important differences between the submitted version and the official published version of record.

People interested in the research are advised to contact the author for the final version of the publication, or visit the DOI to the publisher's website.

- The final author version and the galley proof are versions of the publication after peer review.

- The final published version features the final layout of the paper including the volume, issue and page numbers.

Link to publication

\footnotetext{
General rights rights.

- You may freely distribute the URL identifying the publication in the public portal. please follow below link for the End User Agreement:

www.umlib.nl/taverne-license

Take down policy

If you believe that this document breaches copyright please contact us at:

repository@maastrichtuniversity.nl

providing details and we will investigate your claim.
}

Copyright and moral rights for the publications made accessible in the public portal are retained by the authors and/or other copyright owners and it is a condition of accessing publications that users recognise and abide by the legal requirements associated with these

- Users may download and print one copy of any publication from the public portal for the purpose of private study or research.

- You may not further distribute the material or use it for any profit-making activity or commercial gain

If the publication is distributed under the terms of Article 25fa of the Dutch Copyright Act, indicated by the "Taverne" license above, 


\title{
An in vitro model to evaluate the properties of matrices produced by fibroblasts from osteogenesis imperfecta and Ehlers-Danlos Syndrome patients
}

\author{
Dimitra Micha ${ }^{a, *}$, Gerard Pals a, Theo H. Smit ${ }^{\text {b, c }}$, Samaneh Ghazanfari ${ }^{\text {b, d, e }}$ \\ a Department of Clinical Genetics, Amsterdam Movement Sciences, Amsterdam University Medical Centers, VUmc, Amsterdam, the Netherlands \\ ${ }^{\mathrm{b}}$ Department of Orthopedic Surgery, Amsterdam University Medical Centers, Amsterdam, the Netherlands \\ ${ }^{c}$ Department of Medical Biology, Amsterdam University Medical Centers, Amsterdam, the Netherlands \\ ${ }^{\mathrm{d}}$ Aachen-Maastricht Institute for Biobased Materials, Faculty of Science and Engineering, Maastricht University, Geleen, the Netherlands \\ e Department of Biohybrid \& Medical Textiles (Biotex), RWTH Aachen University, Aachen, Germany
}

\section{A R T I C L E I N F O}

\section{Article history:}

Received 10 September 2019

Accepted 20 September 2019

Available online 24 October 2019

\section{Keywords:}

Collagen

Osteogenesis imperfecta

Ehlers-Danlos syndrome

Extracellular matrix

In vitro disease model

Collagen anisotropy

\begin{abstract}
A B S T R A C T
Aim of the study: Osteogenesis imperfecta and Ehlers Danlos syndrome are hereditary disorders caused primarily by defective collagen regulation. Osteogenesis imperfecta patients were divided to haploinsufficient and dominant negative depending on the effect of COL1A1 and COL1A2 mutations whereas Ehlers Danlos syndrome patients had a mutation in PLOD1. Although collagen abnormalities have been extensively studied in monolayer cultures, there are no reports about 3D in vitro models which may reflect more accurately the dynamic cell environment. This is the first study presenting the structural and mechanical characterization of a 3D cell-secreted model using primary patient fibroblasts.

Materials and methods: Fibroblasts from patients with osteogenesis imperfecta and Ehlers Danlos syndrome were cultured with ascorbic acid for 5 weeks. The effect of mutations on cytosolic and secreted collagen was tested by electrophoresis following incubation with radiolabeled ${ }^{14} \mathrm{C}$ proline. Extracellular matrix was studied in terms of collagen fiber orientation, stiffness, as well as glycosaminoglycan and collagen content.

Results and conclusions: Osteogenesis imperfecta patients with haploinsufficient mutations had higher percentage of anisotropic collagen fibers alignment compared to other patient groups; all patients had a lower percentage of anisotropic samples compared to healthy controls. This correlated with higher average stiffness in the control group. Glycosaminoglycan content was lower in the control and haploinsufficient groups. In cells with PLOD1 mutations, there were no differences in PLOD2 expression. This proof of concept study was able to show differences in collagen fiber orientation between different patient groups which can potentially pave the way towards the development of 3D models aiming at improved investigation of disease mechanisms.
\end{abstract}

(c) 2019 Elsevier Inc. All rights reserved.

\section{Introduction}

Collagen is the most abundant protein in the human body. It is a major structural component of the connective tissue which has a supporting, connecting and protective function in diverse anatomical locations [1]. For this reason, it is easy to deduce that problems related to any aspect of its biosynthesis, regulation and

\footnotetext{
* Corresponding author. Department of Clinical Genetics, Amsterdam University Medical Centers, Van der Boechorststraat 7, 1081 BT, Amsterdam, the Netherlands. E-mail address: d.micha@amsterdamumc.nl (D. Micha).
}

remodeling will have detrimental effects on a multisystemic level leading to distinct clinical conditions. These are collectively referred to as collagenopathies, of which Osteogenesis Imperfecta (OI) and Ehlers-Danlos Syndrome (EDS) are common [2]. Although the genetic defects of these diseases are well described, there is still little information to explain variability in clinical presentation and severity as a result of collagen dysregulation. Appropriate in vitro models in which collagen and physiological cell behavior can be studied in these diseases are lacking and thus present an unmet need in this field.

OI and EDS are inherited connective tissue disorders arising from many different mutations in collagen genes or genes affecting 
the collagen biosynthetic pathway [3]. OI, known as brittle bone disease, is a common syndromic form of hereditary bone fractures; patients experience numerous bone fractures during their lifetime and in severe cases, this is accompanied by serious skeletal deformities. Syndromic features vary and may include blue sclerae, deafness and dentinogenesis imperfecta [4,5]. The central role of collagen in OI is highlighted by the fact that many of the $20 \mathrm{OI}$ causative genes are directly or indirectly implicated in collagen regulation [6]. The vast majority of cases are caused by mutations in the COL1A1 and COL1A2 genes, which are the proteins form collagen type I [7]. The phenotypic effect of the mutation differs based on the molecular defect. Mutations are classified as haploinsufficient (HI), when no collagen is produced by the defective allele, and as dominant negative (DN), when the mutation affects the protein structurally [8]. HI mutations cause a mild clinical picture, typically recognized by bone fragility and blue sclerae, whereas DN mutations can lead to more severe cases with skeletal dysplasia. The focus of EDS pathology is placed on skin fragility, joint hypermobility and vascular manifestations [9]. Similar to OI, genetic causes largely include mutations in collagen genes and collagen-modifying enzymes, which influence the phenotypic outcome $[10,11]$. In particular, mutations in the collagen-regulating gene PLOD1 cause kyphoscoliosis as the main recognizable characteristic [12].

Considering that collagen is highly prevalent throughout the body, it is enigmatic why specific mutations in collagen-related genes would only affect specific organ systems in OI and EDS. In order to understand this, the properties of defective collagens and their associated extracellular matrix (ECM) need to be interrogated in different in vitro models. Quantitative and qualitative analysis of collagen produced by cultured primary dermal fibroblasts has been routinely used to determine the molecular effects of gene mutations [13]. Fibroblasts are the main collagen-producing cells in the skin and they can be acquired by a skin biopsy which is considered a minimally invasive technique [14]. Thus far, in vitro collagen analyses of OI and EDS have been performed with a monolayer 2D culture, which limits the model validity regarding the amount, type and post-translational modification of collagen in the cell and media fractions as it does not allow ECM properties assessment. Therefore, a more physiologically relevant model is required to better simulate the environment of a living diseased tissue and to further study the interaction of the cells and ECM and their structural arrangement.

Fibroblasts are responsible for the production of ECM in connective tissues by secreting collagen, glycosaminoglycans and proteoglycans. Fibrillar collagen occupies the largest percentage of ECM mass. It includes approximately $70 \%$ of the dry mass of the dermis [14], most of which is collagen type I, whereas type III and IV are markedly less abundant but still important determinants of ECM properties $[10,15]$. All collagens are trimeric helices consisting of a long triple helical domain between an $\mathrm{N}$ - and a C-terminus non-triple helical domain; in this helix, two chains are coded by the COL1A1 gene while one is coded by the COL1A2 gene. The three proline-rich collagen chains are produced in the endoplasmic reticulum, where they form the procollagen precursor which is extensively modified post-translationally, especially by hydroxylation. In particular, enzymes of the procollagen-lysine 2oxoglutarate 5-dioxygenase (PLOD) family catalyse the hydroxylation of lysine residues which are important for the stability of collagen fibrils after extracellular cleavage of the $\mathrm{N}$ - and C-terminus non-helical procollagen domains [7].

The production of collagen by the fibroblasts is paramount to the integrity of the ECM. In the skin, homeostasis is achieved by fibroblasts secreting ECM and at the same time remodeling the ECM. Fibroblasts are malleable cells that respond morphologically and behaviorally to their biochemical and mechanical environment. This implies that a monolayer of cells cultured on a stiff cell culture plastic surface will not respond the same as those cultured in a softer 3D environment. Thus, development of suitable alternatives, such as bioengineered and cell-secreted matrices, which can mimic the skin environment, are required [16,17]. This study presents an in vitro model for the investigation of ECM properties produced by OI and EDS fibroblasts. Long culture time in combination with ascorbic acid supplement provided cells the ability to build up a 3D ECM. Collagen fiber orientation, as well as ECM stiffness and composition were assessed in relation to the molecular effect of the mutated genes COL1A1, COL1A2 and PLOD1.

\section{Materials and methods}

\subsection{Genetic analysis}

Skin biopsies were acquired from the inner arm of patients after informed consent according to the medical ethics committee of the VU University. Mutation analysis for the COL1A1, COL1A2 and PLOD1 genes was performed by Sanger sequencing. The Alamut visual interactive software v.2.9.0 was used for the in silico prediction of pathogenicity for the identified genetic variants.

\subsection{In vitro matrix production}

Fibroblast cultures were established from dermal punch biopsies following a 4 week culture period of the biopsy on plastic. Cells were seeded at a density of 70,000 per well of 4-well Nunc ${ }^{\mathrm{TM}}$ Lab-Tek $^{\mathrm{TM}}$ II Chambered Coverglasses with $1.5 \mathrm{~mm}$ borosilicate glass bottom in Ham F10 media supplemented with 10\% fetal calf serum (FCS) and 1\% penicillin/streptomycin (Lifetechnologies, country). The cells were kept in a humidified atmosphere at $37^{\circ} \mathrm{C}$ and $5 \% \mathrm{CO}_{2}$. After overnight cell adherence, the cells were refreshed 2 times per week with the aforementioned media $(0.6 \mathrm{ml} /$ well $)$ supplemented with $50 \mu \mathrm{g} / \mathrm{ml}$ 2-Phospho-L-ascorbic acid trisodium salt (Sigma-Aldrich), a stable ascorbic acid derivative, for 5 weeks during which they produced a three-dimensional ECM. Cells were harvested for RNA isolation before vitamin C treatment and after 5 weeks of treatment.

\section{3. $q P C R$}

RNA was isolated with the NucleoSpin ${ }^{\circledR}$ TriPrep kit (MachereyNagel) according to manufacturer's instruction; after aspirating the cell culture media cells were lysed by scraping into the cell lysis buffer of the kit. RNA quantity and quality was determined by Nanodrop. cDNA was synthesized with the SuperScript ${ }^{\circledR}$ VILO cDNA Synthesis Kit and Master Mix (ThermoFisher Scientific) with 140 ng RNA. qPCR analysis was performed to quantify the mRNA expression of PLOD1 and PLOD2. qPCR reactions were performed in 384-well plates with Lightcycler 480 SYBR Green I mastermix using the Lightcycler 480 PCR system (Roche). Preamplification was accomplished at $95^{\circ} \mathrm{C}$ for $10 \mathrm{~min}$ which was subsequently followed by 45 cycles of denaturation at $95^{\circ} \mathrm{C}$ for $10 \mathrm{sec}$, annealing at $56^{\circ} \mathrm{C}$ for $5 \mathrm{sec}$ and elongation at $72^{\circ} \mathrm{C}$ for $10 \mathrm{sec}$. Relative expression of each target gene was calculated with the LightCycler 480 release 1.5.0 SP4 software (Roche); the expression of the housekeeping gene TBP (TATA box binding protein) was used for normalization. The primer sequences are: PLOD1, 5'-CAAGCCGGAGGACAACCTTT3'and 5'- CCAAGCGCCTGGATCTTGTA-3'; PLOD2, 5'-ACTGTGAAGGTCCTTGGTCA-3' and 5'-ACTTCTTCTGGACCACCAGC- $3^{\prime}$; TBP, $5^{\prime}-$ AGTTCTGGGATTGTACCGCA-3' and $5^{\prime}$ - TCCTCATGATTACCGCAGCA-3'. 


\subsection{Collagen electrophoresis}

Quantitative and qualitative analysis of collagen species was performed by electrophoresis of ${ }^{14} \mathrm{C}$ proline-labeled collagen with adjustments to the previously published protocol [18]. The cells were grown in T25 flasks to confluency and then treated with Basal Medium Eagle (BME) media supplemented with 5\% heatinactivated FCS, 1\% HEPES, $1 \%$ penicillin/streptomycin (Lifetechnologies), $0.037 \mathrm{mg} / \mathrm{ml}$ ascorbic acid and $0.05 \mathrm{mg} / \mathrm{ml} \beta$-aminopropionitrile (BAPN) (Sigma-Aldrich); $0.25 \mathrm{ml}$ of ${ }^{14} \mathrm{C}$ proline solution was added to $8.2 \mathrm{ml}$ of the media $(12.5 \mu \mathrm{Ci})$ and $2 \mathrm{ml}$ per flask and cells were incubated overnight. After collection of the media fraction, cells were lysed in $0.5 \mathrm{M}$ acetic acid with $0.5 \%$ Triton-X100. Collagens in $1 \mathrm{ml}$ of the media fraction were treated with $20 \mu \mathrm{l}$ of $0.5 \mathrm{M}$ pH8 EDTA, $10 \mu \mathrm{l} 5 \mathrm{mg} / \mathrm{ml} \mathrm{N}$-ethylmaleide (NEM) and $10 \mu \mathrm{l} 12 \mathrm{mg} / \mathrm{ml}$ Pefabloc and precipitated overnight at $4{ }^{\circ} \mathrm{C}$ with cold $96 \%$ and for $1 \mathrm{~h}$ with $66 \%$ ethanol solution. Collagens were then solubilized in $0.5 \mathrm{M}$ acetic acid. The cell fraction was processed by centrifuging the cell suspension at $500 \mathrm{~g}$ for $2 \mathrm{~min}$, washing once with Earle's Balanced Salt Solution and incubating at $4{ }^{\circ} \mathrm{C}$ for $2 \mathrm{~h}$ in $0.5 \mathrm{M}$ acetic acid with $0.5 \%$ Triton-X100. Both fractions were digested with pepsin at $0.2 \mu \mathrm{g} / \mu \mathrm{l}$ for $4-6 \mathrm{~h}$ at $20^{\circ} \mathrm{C}$; the reaction was stopped with $0.065 \mu \mathrm{g} / \mu \mathrm{l}$ pepstatin (Sigma-Aldrich). After freeze-drying, the samples were dissolved in $20 \mu \mathrm{l}$ 2X SDS loading buffer (30\% 1.25 M Tris-HCl pH6.8, 20\%v/v 10\% SDS, 20\% glycerol and $0.025 \mathrm{mg} / \mathrm{ml}$ bromophenol blue) and electrophoresis was performed in $11.5 \%$ acryl/bisacryl-urea-polyacrylamide gels at $200 \mathrm{~V}$ and $\sim 110 \mathrm{~mA}$ for $90 \mathrm{~min}$ at $8^{\circ} \mathrm{C}$. In order to allow collagen type III $\alpha$ chains to enter the gel, $30 \mu \mathrm{l}$ of $7.7 \mathrm{mg} / \mathrm{ml}$ DTT in sucrose was added per well. After $5 \mathrm{~min}$ incubation, electrophoresis was continued at $400 \mathrm{~V}$ and $\sim 95 \mathrm{~A}$ for $90 \mathrm{~min}$ at $8^{\circ} \mathrm{C}$. Gels were vacuumdried and visualized by autoradiography. After quantification of the different collagen species COL1, COL3A1 and COL5A1 by autoradiography phosphor imager plates, values for the cytosol and media fraction were determined as a percentage by using the following formulas, respectively:

$\%$ COL1 $=($ COL1A1-background $)+($ COL1A2-background $) /($ COL1A1background $)+($ COL1A2-background $)+($ COL3A1-

background $)+($ COL5A1-background) $) * 100 \%$;

\%COL3A1 $=($ COL3A1-background $) /(($ COL1A1-

background $)+($ COL1A2-background $)+($ COL5A1-background $))^{*} 100$;

\%COL5A1 $=($ COL5A1-background $) /(($ COL1A1-

background $)+($ COL1A2-background $)+($ COL3A1-background $) * 100$

Reference values for these measurements have been established with dermal fibroblasts from a range of 30 healthy individuals: collagen type I 80-95\%; COL3A1 5-20\% (data not shown). The ratio of COL1A1 to COL2A1 was also determined as COL1A1/ COL1A2 $=($ COL1A1-background $) /(\operatorname{COL} 1 \mathrm{~A} 2 /$ background $)$ which is expected to be 2.0 based on the two alpha 1 and one alpha 2 chain stoichiometry in healthy conditions.

\subsection{Collagen crosslinking}

Collagen crosslinking was measured in urine by HPLC as previously described [19]. Measurements showed low ratio of hydroxylysyl pyridinoline to lysyl pyridinoline, which indicates low lysyl hydroxylase activity as a consequence of mutations in PLOD1.

\subsection{Western blotting}

Whole cell lysates of cells cultured for 5 weeks were prepared by lysing cells in NuPAGE $®$ LDS Sample Buffer with NuPAGE $®$ reducing agent. NuPAGE 4-12\% BisTris gels were used to perform electrophoresis. Protein transfer to nitrocellulose was achieved with the iBlot Blotting system. Nitrocellulose membranes were blocked in Odyssey blocking buffer (Westburg) for $1 \mathrm{~h}$ followed by overnight incubation with primary antibodies against PLOD1 (abcam; Cat\#ab171140) and actin (abcam; Cat\#ab14128) in the presence of $0.1 \%$ Triton X-100. Secondary antibody incubation was performed with the IRDye $800 \mathrm{CW}$ goat anti-rabbit IgG and the IRDye $680 \mathrm{CW}$ goat anti-mouse IgG antibodies (LI-COR Biosciences) after which the membranes were scanned with the Odyssey infrared imaging system by using the Odyssey version 4 software (LI-COR Biosciences).

\subsection{Collagen immunostaining and orientation analysis}

Samples were fixed in $4 \%$ formaldehyde after 5 weeks of culture. After fixation, they were incubated at room temperature with blocking buffer (1\% BSA, 20\% donkey serum, PBS) for $1 \mathrm{~h}$ and then with rabbit polyclonal anti-collagen type I antibody (1:1000, Cat\#ab34710, Abcam, USA) for 2 h. The samples were then washed 3 times in PBS. A few drops of Vectashield mounting medium containing DAPI (Vector Laboratories, USA) were added to each well afterwards, and images using an inverted confocal microscope (Leica SP8, Leica, Germany) were taken. Each well was imaged at 5 different locations, and at each location, a stack of about 10 images with a distance of $2 \mu \mathrm{m}$ between adjacent optical slices through the thickness of the samples was obtained. Samples were categorized based on the total number of collagen alignment changes observed through the stack of images: it is called "one direction", if the alignment of collagen fibers stayed the same through the stack of images. If the alignment was changed to a second almost perpendicular direction, the sample was categorized in the "two directions" group, and if the alignment stayed random, it was categorized in the "random" group. Three samples per group were imaged.

\subsection{Nano-indentation}

Surface stiffness of the samples at the end of 5 weeks culture time were measured using PIUMA nano-indentor (PIUMA, Optics11, Amsterdam, The Netherlands). Measurements were perfomed on fresh samples in PBS. A cantilever with a radius of $88 \mu \mathrm{m}$ and a stiffness of $0.85 \mathrm{~N} / \mathrm{m}$ was used to indent the surface for $15 \mu \mathrm{m}$. The loading and unloading time was set to $5 \mathrm{~s}$, and the effective Young's modulus was calculated based on the load and displacement data. Each sample was indented at 5 different locations and the data were averaged per sample.

\subsection{Biochemical analysis}

Three samples per patient and control donor were analysed using biochemical data and represented as an average. Samples were lyophilized and then digested in papain solution $(100 \mathrm{mM}$ phosphate buffer, $5 \mathrm{mM}$ L-cysteine, $5 \mathrm{mM}$ ethylenediaminetetraacetic acid, and $125-140 \mathrm{mg}$ papain per $\mathrm{mL}$ ) at $60^{\circ} \mathrm{C}$ for $16 \mathrm{~h}$. Glycosaminoglycan (GAG) content was assessed as previously described $[20,21]$ in which chondroitin sulfate was measured by using $150 \mathrm{~mL}$ dimethyl methylene blue ( $46 \mathrm{mM}$ dimethyl methylene blue, $40.5 \mathrm{mM}$ glycin, $40.5 \mathrm{mM} \mathrm{NaCl}$, pH 3.0) added to $40 \mathrm{~mL}$ of each sample after which the absorbance was read at $540 \mathrm{~nm}$. Chondroitin sulfate from shark cartilage (Sigma) was used for the standard curve. Finally, the HYP, as a measure of collagen content, was also measured as described before [22,23], and a standard curve prepared from trans-4-hydroxyproline. 


\subsection{Statistics}

Stiffness and biochemical content data are shown as mean \pm standard error of mean. One-way ANOVA, followed by Tukey's multiple comparison post hoc test, was performed to evaluate the difference between different groups. GraphPad Prism software (GraphPad Software, Inc.) was used for statistical analysis. A p-value $<0.05$ was considered significant.

\section{Results}

\subsection{Effect of OI and EDS mutations on collagen species}

In OI patients with mutations in collagen type I genes, the disease is inherited in an autosomal dominant manner, whereas in EDS type VI patients with PLOD1 mutations the disease presents an autosomal recessive inheritance pattern. An overview of the gene mutations as well as patient characteristics is given in Supplemental Table 1. Consistent with the pattern of inheritance, mutations in collagen type I genes are heterozygous, whereas mutations in PLOD1 are in homozygous or compound heterozygous state.

Collagen electrophoresis in $2 \mathrm{M}$ denaturing urea conditions allows the analysis of cellular and secreted collagen types COL1A1, COL1A2, COL3A1 and COL5A1 chains (Supplemental Fig. 1A). HI mutations in COL1A1 were predicted to lead to a premature stop codon within this gene which leads to a null allele. In agreement with the HI condition of one protein-producing gene allele, COL1 and the COL1A1/COL1A2 ratio were decreased compared to the normal collagen values (Supplemental Fig. 1B). DN mutations (patients P4-6) did not alter collagen quantitatively but they did lead to collagen overmodification as a result of delayed triple helix folding during which lysine residues are converted to hydroxylysine by excessive hydroxylation $[24,25]$. The overmodification is visualized by the smeary appearance of COL1A1 and COL1A2 bands, in contrast to the healthy control fibroblasts in which they are absent. All DN cell lines presented decreased cytosolic COL3A1 expression. In fibroblast cells with EDS type VI-causing mutations the COL1A1/ COL1A2 ratio was decreased in the cytosolic fraction. PLOD1 has an essential role in hydroxylating lysyl residues in triple helical collagen type I for the formation of crosslinks in collagen fibrils; this is performed by PLOD2 at the two ends outside the triple helix. Lysyl-pyridinoline and hydroxylysyl-pyridinoline crosslinks are formed after oxidation of specific lysyl- and hydroxylysyl-residues. PLOD1 mutations decrease its activity leading to decreased lysyl hydroxylation affecting the formation of crosslinks. The ratio of lysyl pyridinoline to hydroxylysyl pyridinoline, both of which primarily arise from collagen degradation in bone, was found to be increased in the urine of patients P7 and P9 in agreement with impaired lysyl hydroxylase activity in EDS [26].

\subsection{Collagen fiber alignment}

The orientation of collagen fibers was quantified in the 3D ECM in the different groups of cells and it was classified as one direction, two direction or random depending on collagen fiber alignment in optical sections (Fig. 1A, B, C). As expected, the control group showed higher level of organization, as shown by the increased percentage of one direction collagen fibers, compared to the other groups (Fig. 1D). Cells with HI collagen mutations also presented one direction orientation as predominant, although the percentage was lower compared to the control group. Collagen fiber orientation was mostly present in two directions in the PLOD1 group and it was random in the DN collagen mutation group.

\subsection{ECM surface stiffness}

Although controls tend to be stiffer, there was no significant difference between the average stiffness of the samples from patient and control groups (Fig. 2). However, there was variability
A

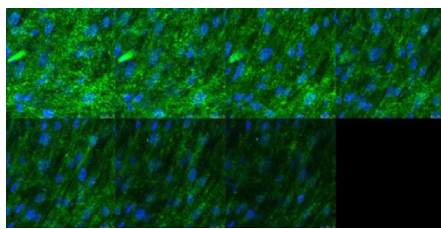

B

$\mathrm{C}$

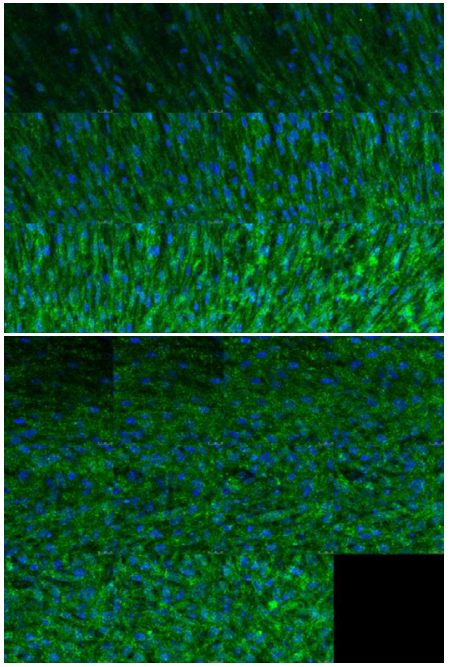

D

\begin{tabular}{lcccc}
\hline & PLOD1 & DN & HI & Control \\
\hline Two directions & $59 \%$ & $35 \%$ & $36 \%$ & $26 \%$ \\
One direction & $23 \%$ & $0 \%$ & $42 \%$ & $74 \%$ \\
Random & $18 \%$ & $65 \%$ & $22 \%$ & $0 \%$ \\
\hline
\end{tabular}

\begin{tabular}{lccc}
\hline \multicolumn{1}{c}{ HI } & P1 & P2 & P3 \\
\hline Two directions & $45 \%$ & $19 \%$ & $45 \%$ \\
One direction & $55 \%$ & $64 \%$ & $9 \%$ \\
Random & $0 \%$ & $17 \%$ & $46 \%$ \\
\hline \multicolumn{1}{c}{ DN } & P4 & P5 & P6 \\
\hline Two directions & $71 \%$ & $34 \%$ & $0 \%$ \\
One direction & $0 \%$ & $0 \%$ & $0 \%$ \\
Random & $29 \%$ & $66 \%$ & $100 \%$ \\
\hline
\end{tabular}

\begin{tabular}{lcccc}
\hline PLOD1 & P7 & P8 & P9 & P10 \\
\hline Two directions & $60 \%$ & $73 \%$ & $40 \%$ & $62 \%$ \\
One direction & $15 \%$ & $18 \%$ & $51 \%$ & $10 \%$ \\
Random & $25 \%$ & $9 \%$ & $9 \%$ & $28 \%$ \\
\hline
\end{tabular}

\begin{tabular}{lccccc}
\hline Control & C1 & C2 & C3 & C4 & C5 \\
\hline Two directions & $45 \%$ & $36 \%$ & $13 \%$ & $28 \%$ & $10 \%$ \\
One direction & $55 \%$ & $64 \%$ & $87 \%$ & $72 \%$ & $90 \%$ \\
Random & $0 \%$ & $0 \%$ & $0 \%$ & $0 \%$ & $0 \%$ \\
\hline
\end{tabular}

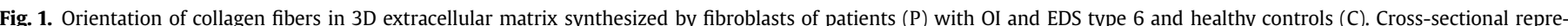

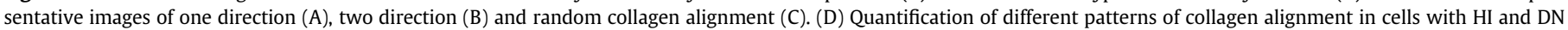
mutations in COL1A1 and COL1A2 and mutations in PLOD1 as well as cells from healthy donors. 

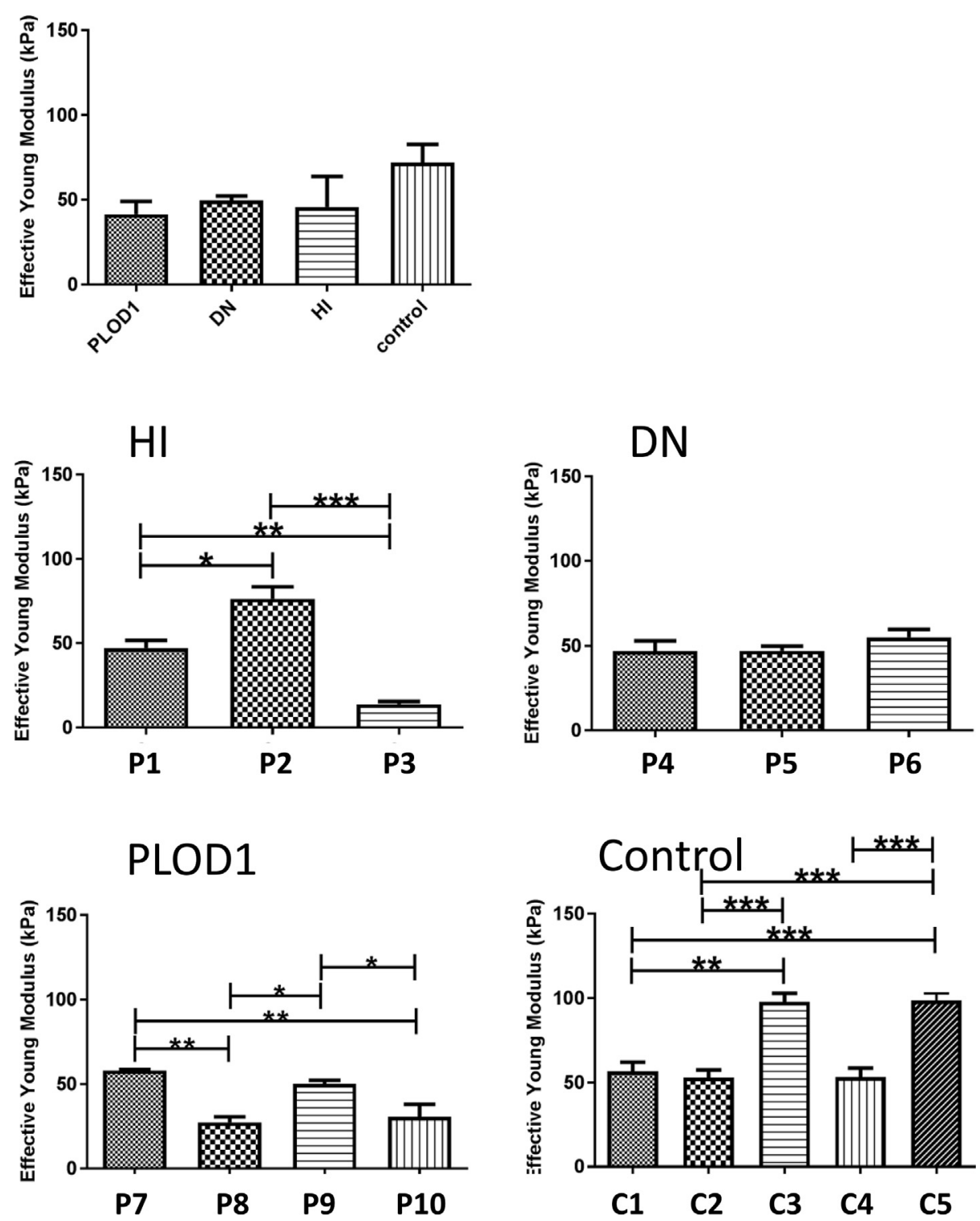

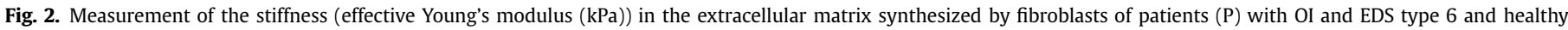

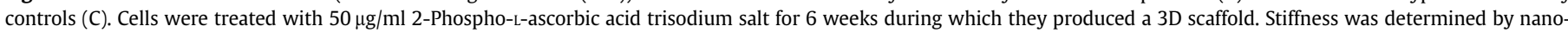

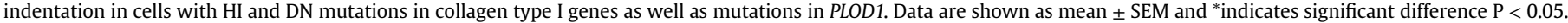

between samples within HI, PLOD1 and control but not DN groups.

\subsection{Biochemical content}

Both the control and HI collagen mutation group showed significantly decreased GAG production compared to the DN group (Fig. 3). Results from HYP measurements showed a low collagen content in the HI group, which was significantly different from the PLOD1 group. These differences were not accounted for by differences in cell proliferation as measured by the DNA content (Supplemental Fig. 2).

\subsection{PLOD expression}

PLOD enzyme exists in three different forms (PLOD1, PLOD2 and PLOD3), all of which are expressed in fibroblasts [27]. PLOD3 differs from the other two forms in that it has glycosylation activity in addition to lysyl hydroxylase activity. The expression of PLOD1 and PLOD2 was investigated to see if PLOD2 is potentially upregulated as a compensation mechanism for the loss of function mutations in PLOD1 which were confirmed to lead to lack of protein expression in the fibroblasts of the 4 EDS type VI patients (Fig. 4A). At the mRNA level, PLOD1 expression was also minimal at the start and after 5 weeks compared to the other groups of cells (Fig. 4B). No upregulation in PLOD2 expression was noted in cells with PLOD1 mutations; PLOD2 expression was increased only in the control cells after 5 week treatment with ascorbate.

\section{Discussion}

Collagen accounts for approximately $70 \%$ of the dry mass in the skin dermis; the rest consists of elastin, and proteoglycans, the latter of which provides a hydrated environment for the cells $[28,29]$. The content of total collagen can be mostly attributed to collagen type I (80-95\%) and III (5-20\%) and to a lesser extent collagen type $\mathrm{V}$ (unpublished data). The high need for collagen production is met by fibroblasts which are the predominant cell type in the dermis [30]. Skin relies on collagen for tensile strength 

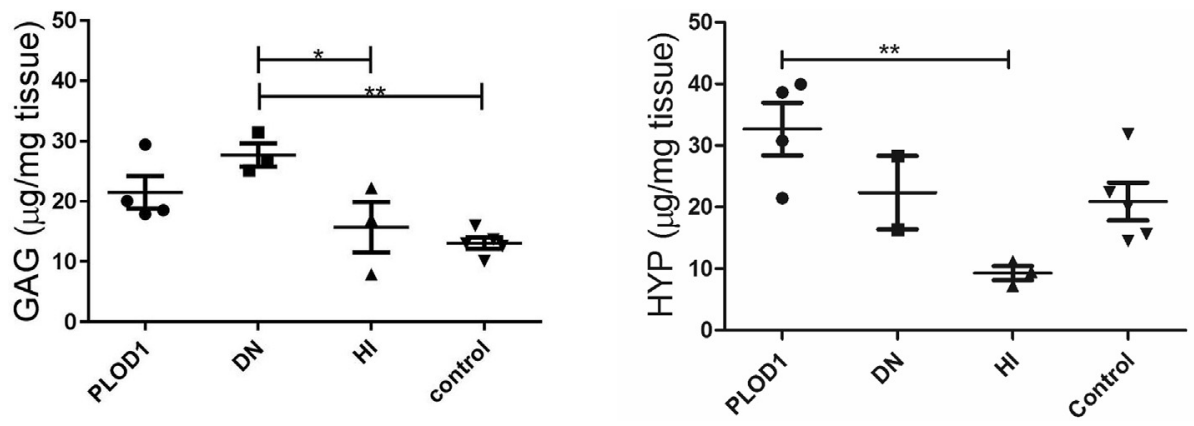

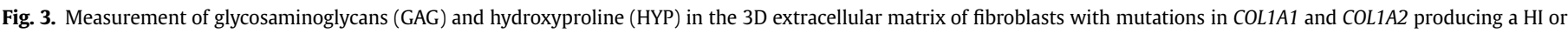
DN effect, mutations in PLOD1 and healthy controls.

A
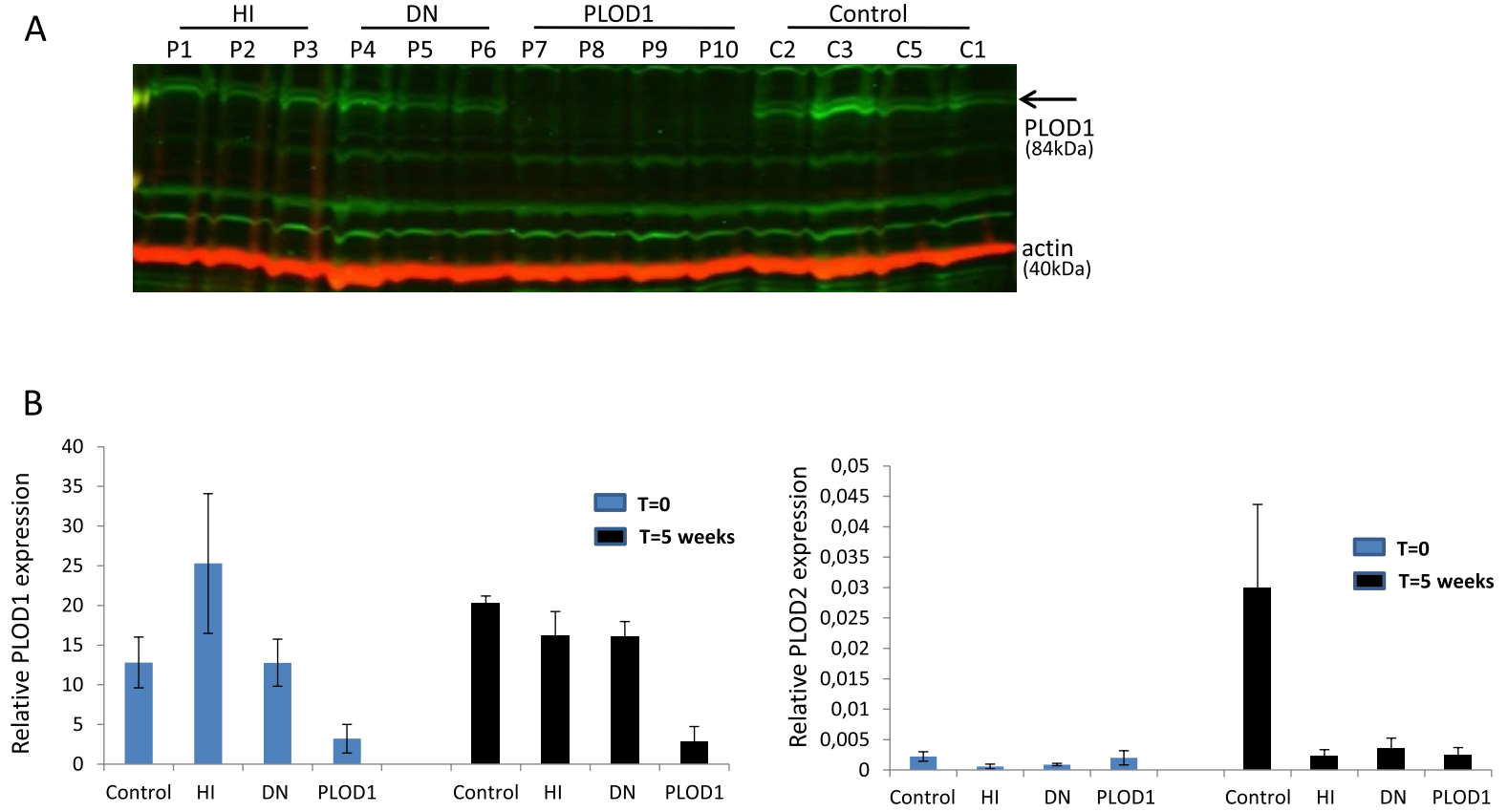

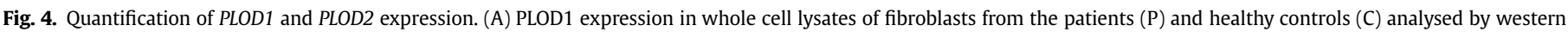

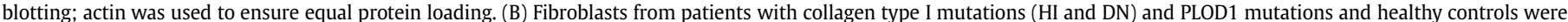

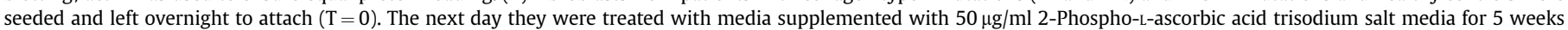

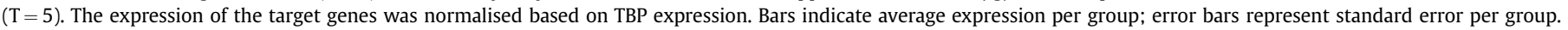

and stiffness for which reason genetic defects affecting the quantity, quality and/or ratio of collagens produce a pathological phenotype in OI and EDS. It is well documented that the skin of OI patients shows thinness, translucency, easy bruisability, and impaired elasticity [31]; decreased elasticity has been also observed compared to healthy counterparts [32]. Quantification of dermal manifestations has been attempted in several studies; quantitative MRI, for example, was able to distinguish between human OI and control skin samples [32]. The morphometric assessment of elastic fibre area fraction and ultrastructural characteristics were also revealed to be abnormal in OI patients [33]. A different study based on nonlinear microscopy methods coupled to image-analysis approaches, identified a reduction in the elastic component of dermis in OI patients but no differences in collagen intensity [34]. However, these studies have been hindered by small sample size and in some cases by lack of genetic characterisation. EDS patients with mutations in PLOD1 (EDS type VI) also show aberrant skin characteristics such as skin hyperextensibility and fragility exhibited by easy bruising and delayed healing [35]. Even though several reports exist about cutaneous measurements in other types of EDS [36,37], this is lacking for type VI.

It is clear from the above that no reliable method still exists for the 3D characterization of skin tissue in OI and EDS type VI patients; furthermore, no physiologically relevant in vitro models are reported for these diseases. Here we developed an in vitro model for the study of collagen and ECM properties with primary fibroblasts from patients with a well described effect of mutations. In this model, it is shown that fibroblasts from the control group have the highest percentage of one direction collagen fibers (74\%) compared to the three patient groups (Fig. 1) which reflects higher level of ECM organization in the healthy condition. The HI group showed higher percentage of one direction collagen fibers compared to the DN and PLOD1 groups. This can be possibly attributed to the production of normal quality collagen (Supplemental Fig. 1), despite decreased quantity which was shown by collagen electrophoresis. The group with PLOD1 mutations had the higher percentage of two 
direction collagen fibers (59\%) while the DN group showed the highest percentage of random orientation (65\%); this correlates with lack of PLOD1 expression whereas no differences were found in PLOD2 expression (Fig. 4). Fibroblasts from the DN group are from patients with mutations in COL1A1 and COL1A2 which are shown to lead to collagen overmodification. In specific molecular defects, such as the replacement of the small amino acid glycine in the triple helix by the bulkier alanine, proper triple helix folding can be delayed, which results in the exposure of the unfolded collagen chains, $\mathrm{N}$-terminal to the mutation, to overmodification by prolyl- and lysyl hydroxylases. Moreover, the severity of the effect of glycine substitution has been also found to correlate with the proximity of the mutation to the C-terminus where folding starts, and thus also with disease severity [38]. Given that the reported glycine substitutions occur approximately in the middle of the collagen helix, this has an overmodifying effect on almost half of the molecule. Overmodification can affect its stability, secretion and crosslinking in the ECM, which may be responsible for the prevalent random fiber organisation in this model [39,40].

The control group was found to have the highest ECM stiffness as indicated by the average effective Young's modulous, although it was not found to be significant (Fig. 2). In the control group there was a lot of variation in stiffness which was not found to correlate to donor age or cell passage number at biopsy acquisition. It has been shown in previous studies that the arrangement of collagen fibers has a huge impact on the mechanical properties of the constructs [22]. We were not able to see differences between EDS type 6 and OI patients, so differences in patient skin mechanical properties are not represented by the effective Young's modulus measurements. The effective Young's modulus also did not correlate with the GAG content in the different groups (Fig. 3). On average, the control and HI groups showed lower GAG production compared to the DN and PLOD1 groups; it is unclear how this relates to the higher percentage of one direction collagen fibers in the control and HI cells. Moreover, GAG production did not correlate with collagen production neither in the conventional plastic or 3D culture. Limited information exists about the GAG content in OI patients which lacks description of genetic background. Hyaluronan production in skin fibroblasts of OI patients with decreased collagen synthesis was found to be higher per cell than in controls; no differences were observed in the production of sulphated glycosaminoglycans [41]. Another study also showed increased GAG to collagen type I ratio in OI fibroblasts [42], whereas reduced content of chondroitin sulfate was observed in the skin of two OI patients [43]. Total skin weight contains $0.1-0.3 \%$ GAGs of which hyaluronan is the predominant one [44] as opposed to chondroitin sulfate which was measured in this study, the role of which remains unclear in this model. The reported differences are not due to differences in cell proliferation as shown by total DNA content (Supplemental Fig. 2). Another limitation of this study is that it does not inform on elastin content which also contributes to tissue elasticity and resilience. It should however be mentioned that elastin is barely produced in in vitro cultures and this is one of the main limitation of in vitro models [20].

Using our proposed in vitro model, the ECM architecture and mechanical properties can be studied in OI and EDS type VI patient fibroblasts. The reciprocal interaction between cells and ECM cannot be effectively simulated in a monolayer cell culture system. The pathology of both disorders depends on collagen defects. Therefore, the study of the collagen-producing fibroblasts has the potential to reveal insights on disease pathology. It is known that fibroblast morphology and behavior is influenced by matrix organization and stiffness to which fibroblasts react by subjecting the matrix to constant remodeling [45].

Considering that the most severe disease presentation is severe osteoporosis, bone fractures and skeletal dysplasia in OI, and kyphoscoliosis in EDS type VI, future efforts will be directed to adjusting this model for the study of bone. We have developed a method with which we can transdifferentiate primary fibroblasts to osteoblast-like cells with human platelet lysate [46,47] which are potentially suitable for the generation of this model. Further exploration of this model can assess to which extend it can complement patient diagnosis. Moreover, skin and bone have similar organic component consistency [43]; thus, understanding of ECM defects in skin may potentially help to shed light on pathological mechanisms in bone tissue diseases which is a necessary step towards the development of meaningful therapies.

\section{Conclusion}

To our knowledge this is the first report on the characterisation of a fibroblast 3D matrix for the study of OI and EDS type VI, both of which are diseases caused by collagen defects. Fibroblasts have been used for decades in traditional monolayer culture for the study of collagen defects in these diseases in an effort to investigate the cause of bone fragility and skeletal deformation in relation to collagen regulation. In this model, we showed differences in collagen fiber orientation and stiffness between patient groups and controls. Future work is required to see how analogous this model is to bone physiology and then adjust the model to a more physiologically relevant environment.

\section{Declaration of competing interestsCOI}

The authors declare no conflict of interest.

\section{Acknowledgements}

We kindly acknowledge the contribution of the patients and healthy donors in providing skin tissue material for this study. The graphical abstract was created with BioRender.com.

\section{Appendix A. Supplementary data}

Supplementary data to this article can be found online at https://doi.org/10.1016/j.bbrc.2019.09.081.

\section{Transparency document}

Transparency document related to this article can be found online at https://doi.org/10.1016/j.bbrc.2019.09.081.

\section{References}

[1] S. Ricard-Blum, The collagen family, Cold Spring Harb. Perspect. Biol. 3 (1) (Jan. 2011) a004978.

[2] R. Jobling, et al., The collagenopathies: review of clinical phenotypes and molecular correlations, Curr. Rheumatol. Rep. 16 (1) (Jan. 2014) 394.

[3] D.H. Cohn, P.H. Byers, Clinical screening for collagen defects in connective tissue diseases, Clin. Perinatol. 17 (4) (Dec. 1990) 793-809.

[4] F.S. Van Dijk, G. Pals, R.R. Van Rijn, P.G.J. Nikkels, J.M. Cobben, Classification of osteogenesis imperfecta revisited, Eur. J. Med. Genet. 53 (1) (Jan. 2010) 1-5.

[5] D.O. Sillence, A. Senn, D.M. Danks, Genetic heterogeneity in osteogenesis imperfecta, J. Med. Genet. 16 (2) (Apr. 1979) 101-116.

[6] S. Tournis, A.D. Dede, Osteogenesis imperfecta - a clinical update, Metabolism 80 (Mar. 2018) 27-37.

[7] S. Ricard-Blum, The collagen family, Cold Spring Harb. Perspect. Biol. 3 (1) (Jan. 2011) a004978.

[8] De Paepe, Heritable collagen disorders: from phenotype to genotype, Verh. K. Acad. Geneeskd. Belg. 60 (5) (1998), pp. 463-482-4.

[9] F. Malfait, R. Wenstrup, A. De Paepe, Classic Ehlers-Danlos Syndrome, University of Washington, Seattle, 1993.

[10] J.R. Mao, J. Bristow, The Ehlers-Danlos syndrome: on beyond collagens, J. Clin. Investig. 107 (9) (May 2001) 1063-1069.

[11] S. D'hondt, T. Van Damme, F. Malfait, Vascular phenotypes in nonvascular 
subtypes of the Ehlers-Danlos syndrome: a systematic review, Genet. Med. 20 (6) (Jun. 2018) 562-573.

[12] H.N. Yeowell, B. Steinmann, PLOD1-Related Kyphoscoliotic Ehlers-Danlos Syndrome, University of Washington, Seattle, 1993.

[13] W.A. Cabral, S. Milgrom, A.D. Letocha, E. Moriarty, J.C. Marini, Biochemical screening of type I collagen in osteogenesis imperfecta: detection of glycine substitutions in the amino end of the alpha chains requires supplementation by molecular analysis, J. Med. Genet. 43 (8) (Aug. 2006) 685-690.

[14] C. Viennet, P. Muret, Fibroblast evaluation: extracellular matrix synthesis, in: Measuring the Skin, Springer International Publishing, Cham, 2015, pp. 1-5.

[15] D.E. Birk, J.M. Fitch, J.P. Babiarz, K.J. Doane, T.F. Linsenmayer, Collagen fibrillogenesis in vitro: interaction of types $\mathrm{I}$ and $\mathrm{V}$ collagen regulates fibril diameter, J. Cell Sci. 95 (Pt 4) (Apr. 1990) 649-657.

[16] K.A. Beningo, Y.-L. Wang, Flexible substrata for the detection of cellular traction forces, Trends Cell Biol. 12 (2) (Feb. 2002) 79-84.

[17] E. Cukierman, R. Pankov, K.M. Yamada, Cell interactions with threedimensional matrices, Curr. Opin. Cell Biol. 14 (5) (Oct. 2002) 633-639.

[18] J. Körkkö, L. Ala-Kokko, A. De Paepe, L. Nuytinck, J. Earley, D.J. Prockop, Analysis of the COL1A1 and COL1A2 genes by PCR amplification and scanning by conformation-sensitive gel electrophoresis identifies only COL1A1 mutations in 15 patients with osteogenesis imperfecta type I: identification of common sequences of null-allele mutations, Am. J. Hum. Genet. 62 (1) (Jan. 1998) 98-110.

[19] U. Schwarze, et al., Mutations in FKBP10, which result in Bruck syndrome and recessive forms of osteogenesis imperfecta, inhibit the hydroxylation of telopeptide lysines in bone collagen, Hum. Mol. Genet. 22 (1) (Jan. 2013) $1-17$.

[20] S. Ghazanfari, et al., In vivo collagen remodeling in the vascular wall of decellularized stented tissue-engineered heart valves, Tissue Eng. A 21 (15-16) (Aug. 2015) 2206-2215.

[21] S. Ghazanfari, A. Driessen-Mol, S.P. Hoerstrup, F.P.T. Baaijens, C.V.C. Bouten, Collagen matrix remodeling in stented pulmonary arteries after transapical heart valve replacement, Cells Tissues Organs 201 (3) (2016) 159-169.

[22] S. Ghazanfari, K.A. Alberti, O. Xu, A. Khademhosseini, Evaluation of an elastic decellularized tendon-derived scaffold for the vascular tissue engineering application, J. Biomed. Mater. Res. A 107 (6) (Feb. 2019) 1225-1234.

[23] G. Huszar, J. Maiocco, F. Naftolin, Monitoring of collagen and collagen fragments in chromatography of protein mixtures, Anal. Biochem. 105 (1) (Jun. 1980) 424-429.

[24] J. Engel, D.J. Prockop, The Zipper-Like Folding Of Collagen Triple Helices And The Effects Of Mutations That Disrupt The Zipper, 1991.

[25] M. Raghunath, P. Bruckner, B. Steinmann, Delayed triple helix formation of mutant collagen from patients with osteogenesis imperfecta, J. Mol. Biol. 236 (3) (Feb. 1994) 940-949.

[26] B. Steinmann, D.R. Eyre, P. Shao, Urinary pyridinoline cross-links in EhlersDanlos syndrome type VI, Am. J. Hum. Genet. 57 (6) (Dec. 1995) 1505-1508.

[27] Y. Qi, R. Xu, Roles of PLODs in collagen synthesis and cancer progression, Front. Cell Dev. Biol. 6 (Jun. 2018) 66.

[28] M.D. Shoulders, R.T. Raines, Collagen structure and stability, Annu. Rev. Biochem. 78 (2009) 929-958.

[29] J.E. Fitzpatrick, J.G. Morelli, Dermatology Secrets Plus, Elsevier/Mosby, 2011.

[30] J.M. Sorrell, A.I. Caplan, Fibroblast heterogeneity: more than skin deep, J. Cell
Sci. 117 (Pt5) (2004) 667-675.

[31] L. Chien, E.W. Mu, S. Kang, Skin in osteogenesis imperfecta, Osteogenes Imperfecta (Jan. 2014) 283-288.

[32] Hansen, G.B.E. Jemec, The mechanical properties of skin in osteogenesis imperfecta, Arch. Dermatol. 138 (7) (Jul. 2002) 909-911.

[33] M. Balasubramanian, et al., Ultrastructural and histological findings on examination of skin in osteogenesis imperfecta, Clin. Dysmorphol. 24 (2) (Apr. 2015) 45-54.

[34] J. Adur, et al., The severity of osteogenesis imperfecta and type I collagen pattern in human skin as determined by nonlinear microscopy: proof of principle of a diagnostic method, PLoS One 8 (7) (Jul. 2013), e69186.

[35] H.N. Yeowell, B. Steinmann, PLOD1-Related Kyphoscoliotic Ehlers-Danlos Syndrome, University of Washington, Seattle, 1993.

[36] C. Eisenbeiss, A. Martinez, M. Hagedorn-Greiwe, D.P. Reinhardt, B. Bätge, J. Brinckmann, Reduced skin thickness: a new minor diagnostic criterion for the classical and hypermobility types of Ehlers-Danlos syndrome, Br. J. Dermatol. 149 (4) (Oct. 2003) 850-852.

[37] C. Catala-Pétavy, L. Machet, G. Georgesco, F. Pétavy, A. Maruani, L. Vaillant, Contribution of skin biometrology to the diagnosis of the Ehlers-Danlos syndrome in a prospective series of 41 patients, Skin Res. Technol. 15 (4) (Nov. 2009) 412-417.

[38] J. Xiao, H. Cheng, T. Silva, J. Baum, B. Brodsky, Osteogenesis imperfecta missense mutations in collagen: structural consequences of a glycine to alanine replacement at a highly charged site, Biochemistry 50 (50) (Dec. 2011) $10771-10780$.

[39] M. Raghunath, P. Bruckner, B. Steinmann, Delayed triple helix formation of mutant collagen from patient with osteogenesis imperfecta, J. Mol. Biol. 236 (3) (Feb. 1994) 940-949.

[40] T. Baker, J.A. Ramshaw, D. Chan, W.G. Cole, J.F. Bateman, Changes in collagen stability and folding in lethal perinatal osteogenesis imperfecta. The effect of alpha 1 (I)-chain glycine-to-arginine substitutions, Biochem. J. 261 (1) (Jul. 1989) 253-257.

[41] H. Turakainen, Altered glycosaminoglycan production in cultured osteogenesis-imperfecta skin fibroblasts, Biochem. J. 213 (1) (Jul. 1983) $171-178$.

[42] J. Nazaruk Galicka, M. Bruczko, Differential effect of flavonoids on glycosaminoglycan content and distribution in skin fibroblasts of patients with type I osteogenesis imperfecta, Mol. Med. Rep. 3 (3) (Apr. 2010) 537-541.

[43] G. Cetta, L. Lenzi, M. Rizzotti, A. Ruggeri, M. Valli, M. Boni, Osteogenesis imperfecta: morphological, histochemical and biochemical aspects. Modifications induced by (+)-catechin, Connect. Tissue Res. 5 (1) (1977) 51-58.

[44] J. Salbach, et al., Regenerative potential of glycosaminoglycans for skin and bone, J. Mol. Med. 90 (6) (Jun. 2012) 625-635.

[45] S. Rhee, Fibroblasts in three dimensional matrices: cell migration and matrix remodeling, Exp. Mol. Med. 41 (12) (Dec. 2009) 858

[46] N. Miyake, et al., X-linked hypomyelination with spondylometaphyseal dysplasia (H-SMD) associated with mutations in AIFM1, Neurogenetics 18 (4) (Dec. 2017) 185-194.

[47] D. Micha, et al., Inhibition of TGF $\beta$ signaling decreases osteogenic differentiation of fibrodysplasia ossificans progressiva fibroblasts in a novel in vitro model of the disease, Bone 84 (Mar. 2016) 169-180. 